\title{
REGIONS OF VARIABILITY FOR FUNCTIONS OF BOUNDED DERIVATIVES
}

\author{
HiROSHI YANAGIHARA
}

To the memory of Professor Nobuyuki Suita

\section{Introduction}

For a complex number $\alpha$ with $|\alpha| \leq 1$ let $\mathscr{B}(\alpha)$ be the class of analytic functions $f$ in the unit disc $\mathbf{D}$ with $f(0)=f^{\prime}(0)-\alpha=0$ satisfying $\left|f^{\prime}(z)\right| \leq 1$ in D. Similarly for $\operatorname{Re} \alpha>0$ let $\mathscr{P}(\alpha)$ be the class of analytic functions $f$ in $\mathbf{D}$ with $f(0)=f^{\prime}(0)-\alpha=0$ satisfying $\operatorname{Re} f^{\prime}(z)>0$ in $\mathbf{D}$. For each $z_{0} \in \mathbf{D}$ let

$$
\begin{array}{ll}
V_{\mathscr{B}}\left(z_{0}, \alpha\right)=\left\{f\left(z_{0}\right): f \in \mathscr{B}(\alpha)\right\} & \text { for } \alpha \in \overline{\mathbf{D}}, \\
V_{\mathscr{P}}\left(z_{0}, \alpha\right)=\left\{f\left(z_{0}\right): f \in \mathscr{P}(\alpha)\right\} & \text { for } \operatorname{Re} \alpha>0 .
\end{array}
$$

In this paper we shall determine the variability regions $V_{\mathscr{B}}\left(z_{0}, \alpha\right)$ and $V_{\mathscr{P}}\left(z_{0}, \alpha\right)$ explicitly.

THEOREM 1. If $z_{0}=0$ or $|\alpha|=1$, then $V_{\mathscr{B}}\left(z_{0}, \alpha\right)=\left\{\alpha z_{0}\right\}$. If $z_{0} \neq 0$ and $|\alpha|<1$, then $V_{\mathscr{B}}\left(z_{0}, \alpha\right)$ is the convex closed Jordan domain surrounded by the simple closed curve $\partial \mathbf{D} \ni c \mapsto f_{c}\left(z_{0}\right)$, where

$$
f_{c}(z)=\int_{0}^{z} \frac{c \zeta+\alpha}{1+\bar{\alpha} c \zeta} d \zeta=\frac{z}{\bar{\alpha}}-\frac{1-|\alpha|^{2}}{\bar{\alpha}^{2} c} \log (1+\bar{\alpha} c z), \quad z \in \mathbf{D} .
$$

Furthermore if $f\left(z_{0}\right)=f_{c}\left(z_{0}\right)$ for some $f \in \mathscr{B}(\alpha)$ and $c \in \partial \mathbf{D}$, then $f=f_{c}$. follows.

As a simple application of Theorem 1 we have the sharp growth estimate as

Corollary 2. Let $\alpha, z_{0} \in \mathbf{D} \backslash\{0\}$. Then for $f \in \mathscr{B}(\alpha)$,

$$
\left|f\left(z_{0}\right)\right| \leq\left|f_{c_{0}}\left(z_{0}\right)\right|=\frac{\left|z_{0}\right|}{|\alpha|}-\frac{\left(1-|\alpha|^{2}\right)}{|\alpha|^{2}} \log \left(1+|\alpha|\left|z_{0}\right|\right),
$$

where $c_{0}=\alpha \overline{z_{0}} /\left(|\alpha|\left|z_{0}\right|\right)$ with equality if and only if $f=f_{c_{0}}$.

Received March 25, 2004; revised September 24, 2004. 
TheOREM 3. Let $z_{0} \in \mathbf{D}$ and $\operatorname{Re} \alpha>0$. If $z_{0}=0$, then $V_{\mathscr{P}}\left(z_{0}, \alpha\right)=\{0\}$. If $z_{0} \neq 0$, then $V_{\mathscr{P}}\left(z_{0}, \alpha\right)$ is the convex closed Jordan domain surrounded by the simple closed curve $\partial \mathbf{D} \ni c \mapsto \tilde{f}_{c}\left(z_{0}\right)$, where

$$
\tilde{f}_{c}(z)=\int_{0}^{z}\left(\frac{\alpha+\bar{\alpha} c \zeta}{1-c \zeta}\right) d \zeta=-\bar{\alpha} z+\frac{2 \operatorname{Re} \alpha}{c} \log \frac{1}{1-c z}, \quad z \in \mathbf{D} .
$$

Furthermore if $f\left(z_{0}\right)=\tilde{f}_{c}\left(z_{0}\right)$ for some $f \in \mathscr{P}(\alpha)$ and $c \in \partial \mathbf{D}$, then $f=\tilde{f}_{c}$.

Corollary 4. For $z_{0} \in \mathbf{D} \backslash\{0\}$ and $f \in \mathscr{P}(\alpha)$ we have

$$
\operatorname{Re} \alpha\left\{\frac{2}{\left|z_{0}\right|} \log \left(1+\left|z_{0}\right|\right)-1\right\} \leq \operatorname{Re}\left(\frac{f\left(z_{0}\right)}{z_{0}}\right)
$$

with equality if and only if $f=\tilde{f}_{-\bar{z}_{0} /\left|z_{0}\right|}$. Also we have

$$
\operatorname{Re}\left(\frac{f\left(z_{0}\right)}{z_{0}}\right) \leq \operatorname{Re} \alpha\left\{\frac{2}{\left|z_{0}\right|} \log \frac{1}{1-\left|z_{0}\right|}-1\right\}
$$

with equality if and only if $f=\tilde{f}_{\bar{z}_{0} /\left|z_{0}\right|}$.

Let $S^{*}$ be the class of analytic functions $f$ in $\mathbf{D}$ with $f(0)=f^{\prime}(0)-1=0$ which map D conformally onto a starlike domain with respect to the origin. A function $f \in S^{*}$ is said to be univalent starlike. For a positive integer $p$ let $\left(S^{*}\right)^{p}=\left\{f=f_{0}^{p}: f_{0} \in S^{*}\right\}$. The proofs of Theorems 1 and 3 heavily rely on the following.

LEMMA 5. Let $f$ be an analytic function in $\mathbf{D}$ with $f(z)=z^{p}+\cdots$. If

$$
\operatorname{Re}\left(z \frac{f^{\prime \prime}(z)}{f^{\prime}(z)}\right)>-1, \quad z \in \mathbf{D},
$$

then $f \in\left(S^{*}\right)^{p}$.

Although we could not find any references for proofs of the above lemma, it might be well known. See [3] and [1]. For completeness, by making use of Libera's lemma (see [4] and [2]), we shall give an analytic proof of Lemma 5 in Section 3.

\section{Regions of variability}

In this section, assuming Lemma 5 for the moment, we prove the theorems and corollaries.

Proof of Theorem 1. Clearly we have $V_{\mathscr{B}}(0, \alpha)=\{0\}=\{\alpha \cdot 0\}$. Let $f \in$ $\mathscr{B}(\alpha)$. If $|\alpha|=1$, then from the maximum modulus theorem we have $f(z) \equiv \alpha z$ and hence $V_{\mathscr{B}}\left(z_{0}, \alpha\right)=\left\{\alpha z_{0}\right\}$. 
Suppose that $z_{0} \in \mathbf{D} \backslash\{0\}$ and $|\alpha|<1$. We shall show that $\partial V_{\mathscr{B}}\left(z_{0}, \alpha\right)$ is a simple closed curve and $V_{\mathscr{B}}\left(z_{0}, \alpha\right)$ is the closed domain surrounded by $\partial V_{\mathscr{B}}\left(z_{0}, \alpha\right)$. To this end, since $V_{\mathscr{B}}\left(z_{0}, \alpha\right)$ is a compact and convex subset of $\mathbf{C}$, it suffices to show $V_{\mathscr{B}}\left(z_{0}, \alpha\right)$ contains an open set.

For $|c| \leq 1$ and $z \in \mathbf{D}$ put

$$
f_{c}(z)=\int_{0}^{z} \frac{c \zeta+\alpha}{1+\bar{\alpha} c \zeta} d \zeta=\frac{z}{\bar{\alpha}}-\frac{1-|\alpha|^{2}}{\bar{\alpha}^{2} c} \log (1+\bar{\alpha} c z) .
$$

Then it is easy to see that $f_{c} \in \mathscr{B}(\alpha)$. Since for any fixed $z_{0} \in \mathbf{D} \backslash\{0\}$, the function $\mathbf{D} \ni c \mapsto f_{c}\left(z_{0}\right)$ is nonconstant analytic, it is an open mapping. Thus $V_{\mathscr{B}}\left(z_{0}, \alpha\right)$ contains the open set $\left\{f_{c}\left(z_{0}\right):|c|<1\right\}$.

Next we shall show that $f_{c}\left(z_{0}\right) \in \partial V_{\mathscr{B}}\left(z_{0}, \alpha\right)$ for all $c \in \partial \mathbf{D}$. For $f \in \mathscr{B}(\alpha)$ we have from Schwarz's lemma

$$
\left|\frac{f^{\prime}(z)-\alpha}{1-\bar{\alpha} f^{\prime}(z)}\right| \leq|z|
$$

which is equivalent to

$$
\left|f^{\prime}(z)-\frac{\alpha\left(1-|z|^{2}\right)}{1-|\alpha|^{2}|z|^{2}}\right| \leq \frac{\left(1-|\alpha|^{2}\right)|z|}{1-|\alpha|^{2}|z|^{2}} .
$$

Thus for any $C^{1}$-curve $\gamma: z=z(t), 0 \leq t \leq 1$, with $z(0)=0$ and $z(1)=z_{0}$ we have

$$
\begin{aligned}
\mid f\left(z_{0}\right) & -\int_{0}^{1} \frac{\alpha\left(1-|z(t)|^{2}\right)}{1-|\alpha|^{2}|z(t)|^{2}} z^{\prime}(t) d t \mid \\
& =\left|\int_{0}^{1}\left\{f^{\prime}(z(t))-\frac{\alpha\left(1-|z(t)|^{2}\right)}{1-|\alpha|^{2}|z(t)|^{2}}\right\} z^{\prime}(t) d t\right| \\
& \leq \int_{0}^{1}\left|f^{\prime}(z(t))-\frac{\alpha\left(1-|z(t)|^{2}\right)}{1-|\alpha|^{2}|z(t)|^{2} \mid}\right| z^{\prime}(t) \mid d t \\
& \leq \int_{0}^{1} \frac{\left(1-|\alpha|^{2}\right)|z(t)|}{1-|\alpha|^{2}|z(t)|^{2}}\left|z^{\prime}(t)\right| d t .
\end{aligned}
$$

This implies $f\left(z_{0}\right) \in \overline{\mathbf{D}}(C(\alpha, \gamma), R(\alpha, \gamma))=\{z \in \mathbf{C}:|z-C(\alpha, \gamma)| \leq R(\alpha, \gamma)\}$, where

$$
\begin{aligned}
& C(\alpha, \gamma)=\int_{0}^{1} \frac{\alpha\left(1-|z(t)|^{2}\right)}{1-|\alpha|^{2}|z(t)|^{2}} z^{\prime}(t) d t, \\
& R(\alpha, \gamma)=\int_{0}^{1} \frac{\left(1-|\alpha|^{2}\right)|z(t)|}{1-|\alpha|^{2}|z(t)|^{2}}\left|z^{\prime}(t)\right| d t .
\end{aligned}
$$

Thus we have $V_{\mathscr{B}}\left(z_{0}, \alpha\right) \subset \overline{\mathbf{D}}(C(\alpha, \gamma), R(\alpha, \gamma))$. 
While we have for $|c|=1$

$$
\begin{aligned}
f_{c}^{\prime}(z) & -\frac{\alpha\left(1-|z|^{2}\right)}{1-|\alpha|^{2}|z|^{2}} \\
& =\frac{\left(1-|\alpha|^{2}\right) z(c+\alpha \bar{z})}{\left(1-|\alpha|^{2}|z|^{2}\right)(1+\bar{\alpha} c z)} \\
& =\frac{c\left(1-|\alpha|^{2}\right)}{1-|\alpha|^{2}|z|^{2}} \frac{|z| g(z)}{|g(z)|}
\end{aligned}
$$

where

$$
g(z)=\frac{z}{(1+\bar{\alpha} c z)^{2}}
$$

Since for $z \in \mathbf{D}$

$$
\operatorname{Re}\left(z \frac{g^{\prime}(z)}{g(z)}\right)=\operatorname{Re}\left(\frac{1-\alpha c z}{1+\alpha c z}\right)>0,
$$

by Lemma 5 we have $G(z)=2 \int_{0}^{z} g(\zeta) d \zeta \in\left(S^{*}\right)^{2}$. Thus there exists $G_{0} \in S^{*}$ such that $G=G_{0}^{2}$. Put $\gamma_{0}: z=z(t)=G_{0}^{-1}\left(t^{1 / 2} G_{0}\left(z_{0}\right)\right), 0 \leq t \leq 1$. Then we have $G(z(t))=t G\left(z_{0}\right)$ and hence $2 g(z(t)) z^{\prime}(t)=G^{\prime}(z(t)) z^{\prime}(t)=G\left(z_{0}\right)$. Thus from (7) we have

$$
\begin{aligned}
\left(f_{c}^{\prime}(z(t))-\frac{\alpha\left(1-|z(t)|^{2}\right)}{1-|\alpha|^{2}|z(t)|^{2}}\right) z^{\prime}(t) & =\frac{c\left(1-|\alpha|^{2}\right)|z(t)|}{1-|\alpha|^{2}|z(t)|^{2}} \frac{g(z(t)) z^{\prime}(t)}{|g(z(t))|} \\
& =\frac{c G\left(z_{0}\right)}{\left|G\left(z_{0}\right)\right|} \frac{\left(1-|\alpha|^{2}\right)|z(t)|}{1-|\alpha|^{2}|z(t)|^{2}}\left|z^{\prime}(t)\right|
\end{aligned}
$$

and

$$
\begin{aligned}
f_{c}\left(z_{0}\right)-C\left(\alpha, \gamma_{0}\right) & =\frac{c G\left(z_{0}\right)}{\left|G\left(z_{0}\right)\right|} \int_{0}^{1} \frac{\left(1-|\alpha|^{2}\right)|z(t)|}{1-|\alpha|^{2}|z(t)|^{2}}\left|z^{\prime}(t)\right| d t \\
& =\frac{c G\left(z_{0}\right)}{\left|G\left(z_{0}\right)\right|} R\left(\alpha, \gamma_{0}\right) .
\end{aligned}
$$

Specially we have $f_{c}\left(z_{0}\right) \in \partial \mathbf{D}\left(C\left(\alpha, \gamma_{0}\right), R\left(\alpha, \gamma_{0}\right)\right)$. Since $f_{c}\left(z_{0}\right) \in V_{\mathscr{B}}\left(z_{0}, \alpha\right) \subset$ $\overline{\mathbf{D}}\left(C\left(\alpha, \gamma_{0}\right), R\left(\alpha, \gamma_{0}\right)\right)$, we have $f_{c}\left(z_{0}\right) \in \partial V_{\mathscr{B}}\left(z_{0}, \alpha\right)$.

We deal with uniqueness and show the injectivity of the mapping $\partial \mathbf{D} \ni c \mapsto$ $f_{c}\left(z_{0}\right)$. Suppose that $f\left(z_{0}\right)=f_{c}\left(z_{0}\right)$ for some $f \in \mathscr{B}(\alpha)$ and $c \in \partial \mathbf{D}$. Put

$$
k(t)=\frac{\left|G\left(z_{0}\right)\right|}{c G\left(z_{0}\right)}\left(f^{\prime}(z(t))-\frac{\alpha\left(1-|z(t)|^{2}\right)}{1-|\alpha|^{2}|z(t)|^{2}}\right) z^{\prime}(t) .
$$

Then $k(t)$ is a continuous function of $t \in[0,1]$. And we have from (4) and (9) 


$$
\begin{aligned}
\operatorname{Re} k(t) \leq|k(t)| & \leq \frac{\left(1-|\alpha|^{2}\right)|z(t)|}{1-|\alpha|^{2}|z(t)|^{2}}\left|z^{\prime}(t)\right| \\
& =\frac{\left|G\left(z_{0}\right)\right|}{c G\left(z_{0}\right)}\left(f_{c}^{\prime}(z(t))-\frac{\alpha\left(1-|z(t)|^{2}\right)}{1-|\alpha|^{2}|z(t)|^{2}}\right) z^{\prime}(t) .
\end{aligned}
$$

Thus we have from (9) and (10)

$$
\begin{aligned}
R\left(\alpha, \gamma_{0}\right) & =\operatorname{Re}\left\{\frac{\left|G\left(z_{0}\right)\right|}{c G\left(z_{0}\right)}\left(f_{c}\left(z_{0}\right)-C\left(\alpha, \gamma_{0}\right)\right)\right\} \\
& =\operatorname{Re}\left\{\frac{\left|G\left(z_{0}\right)\right|}{c G\left(z_{0}\right)}\left(f\left(z_{0}\right)-C\left(\alpha, \gamma_{0}\right)\right)\right\} \\
& =\int_{0}^{1} \operatorname{Re} k(t) d t \\
& \leq \int_{0}^{1} \frac{\left|G\left(z_{0}\right)\right|}{c G\left(z_{0}\right)}\left(f_{c}^{\prime}(z(t))-\frac{\alpha\left(1-|z(t)|^{2}\right)}{1-|\alpha|^{2}|z(t)|^{2}}\right) z^{\prime}(t) d t \\
& =R\left(\alpha, \gamma_{0}\right) .
\end{aligned}
$$

This implies

$$
k(t)=\frac{\left|G\left(z_{0}\right)\right|}{c G\left(z_{0}\right)}\left(f_{c}^{\prime}(z(t))-\frac{\alpha\left(1-|z(t)|^{2}\right)}{1-|\alpha|^{2}|z(t)|^{2}}\right) z^{\prime}(t) .
$$

for all $t \in[0,1]$. Hence we have $f^{\prime}=f_{c}^{\prime}$ on $\gamma_{0}$. From the identity theorem for analytic functions we have $f^{\prime}=f_{c}^{\prime}$ and hence $f=f_{c}$ by normalization.

Finally suppose that the mapping $\partial \mathbf{D} \ni c \mapsto f_{c}\left(z_{0}\right)$ is not injective. Then there exist $c_{1}, c_{2} \in \partial \mathbf{D}$ with $c_{1} \neq c_{2}$ such that $f_{c_{1}}\left(z_{0}\right)=f_{c_{2}}\left(z_{0}\right)$. Since $f_{c_{1}}, f_{c_{2}} \in$ $\mathscr{B}(\alpha)$, we have $f_{c_{1}}=f_{c_{2}}$ from uniqueness. This contradicts $c_{1} \neq c_{2}$.

We have shown that the simple closed curve $\partial V_{\mathscr{B}}\left(z_{0}, \alpha\right)$ contains the curve $\partial \mathbf{D} \ni c \mapsto f_{c}\left(z_{0}\right)$. Since a simple closed curve can not contain any simple closed curve other than itself, $\partial V_{\mathscr{B}}\left(z_{0}, \alpha\right)$ coincides with the curve $\partial \mathbf{D} \ni c \mapsto f_{c}\left(z_{0}\right)$.

Proof of Corollary 2. For $w, \beta \in \mathbf{D} \backslash\{0\}$ we have

$$
\left|\frac{w+\beta}{1+\bar{\beta} w}\right| \leq \frac{|w|+|\beta|}{1+|\beta||w|}
$$

with equality if and only if $\bar{\beta} w$ is positive. Thus for $|c|=1$ we have

$$
\begin{aligned}
\left|f_{c}\left(z_{0}\right)\right| & =\left|z_{0} \int_{0}^{1} \frac{c z_{0} t+\alpha}{1+\bar{\alpha} c z_{0} t} d t\right| \\
& \leq\left|z_{0}\right| \int_{0}^{1} \frac{\left|z_{0}\right| t+|\alpha|}{1+|\alpha|\left|z_{0}\right| t} d t=\frac{\left|z_{0}\right|}{|\alpha|}-\frac{1-|\alpha|^{2}}{|\alpha|^{2}} \log \left(1+|\alpha|\left|z_{0}\right|\right)=\left|f_{c_{0}}\left(z_{0}\right)\right|
\end{aligned}
$$


with equality if and only if $c=c_{0}=\alpha \overline{z_{0}} /\left(|\alpha|\left|z_{0}\right|\right)$. Combining this and Theorem 1 we have for any $f \in \mathscr{B}(\alpha)$

$$
\left|f\left(z_{0}\right)\right| \leq \max _{|c|=1}\left|f_{c}\left(z_{0}\right)\right|=\left|f_{c_{0}}\left(z_{0}\right)\right|
$$

with equality if and only if $f=f_{c_{0}}$.

Our proof of Theorem 3 is quite similar to that of Theorem 1. We only outline the proof, details of which may be supplied by the interested reader.

Proof of Theorem 3. Clearly $V_{\mathscr{P}}(0, \alpha)=\{0\}$. Suppose $z_{0} \in \mathbf{D} \backslash\{0\}$. It is easy to see that $V_{\mathscr{P}}\left(z_{0}, \alpha\right)$ is compact and convex subset of $\mathbf{C}$. For $|c| \leq 1$ put

$$
\tilde{f}_{c}(z)=\int_{0}^{z} \frac{\alpha+\bar{\alpha} c \zeta}{1-c \zeta} d \zeta=-\bar{\alpha} z+\frac{2(\operatorname{Re} \alpha)}{c} \log \frac{1}{1-c z} \in \mathscr{P}(\alpha) .
$$

Since the mapping $\mathbf{D} \ni c \mapsto \tilde{f}_{c}\left(z_{0}\right)$ is open, $V_{\mathscr{P}}\left(z_{0}, \alpha\right)$ contains the nonempty open set $\left\{\tilde{f}_{c}\left(z_{0}\right):|c|<1\right\}$. Thus $V_{\mathscr{P}}\left(z_{0}, \alpha\right)$ is a closed Jordan domain surrounded by the simple closed curve $\partial V_{\mathscr{P}}\left(z_{0}, \alpha\right)$.

Let $f \in \mathscr{P}(\alpha)$. From the Schwarz's lemma we have

$$
\left|\frac{f^{\prime}(z)-\alpha}{f^{\prime}(z)+\bar{\alpha}}\right| \leq|z|
$$

which is equivalent to

$$
\left|f^{\prime}(z)-\frac{\alpha+\bar{\alpha}|z|^{2}}{1-|z|^{2}}\right| \leq \frac{2(\operatorname{Re} \alpha)|z|}{1-|z|^{2}}
$$

This implies

$$
V_{\mathscr{P}}\left(z_{0}, \alpha\right) \subset \overline{\mathbf{D}}(\tilde{C}(\alpha, \gamma), \tilde{R}(\alpha, \gamma)),
$$

where $\gamma: z(t), 0 \leq t \leq 1$, is any $C^{1}$-curve with $z(0)=0, z(1)=z_{0}$ and

$$
\begin{aligned}
& \tilde{C}(\alpha, \gamma)=\int_{0}^{1} \frac{\alpha+\bar{\alpha}|z(t)|^{2}}{1-|z(t)|^{2}} z^{\prime}(t) d t, \\
& \tilde{R}(\alpha, \gamma)=\int_{0}^{1} \frac{2(\operatorname{Re} \alpha)|z(t)|}{1-|z(t)|^{2}}\left|z^{\prime}(t)\right| d t .
\end{aligned}
$$

While we have

$$
\tilde{f}_{c}^{\prime}(z)-\frac{\alpha+\bar{\alpha}|z|^{2}}{1-|z|^{2}}=\frac{2(\operatorname{Re} \alpha) c|z|}{1-|z|^{2}} \frac{\tilde{g}(z)}{|\tilde{g}(z)|},
$$

where 


$$
\tilde{g}(z)=\frac{z}{(1-c z)^{2}} .
$$

Since $\operatorname{Re}\left(z \tilde{g}^{\prime}(z) / \tilde{g}(z)\right)=\operatorname{Re}\left\{(1+c z)(1-c z)^{-1}\right\}>0$ for $|z|<1$, there exists $\tilde{G}_{0} \in S^{*}$ satisfying $\tilde{G}(z)=2 \int_{0}^{z} g(\zeta) d \zeta=\tilde{G}_{0}(z)^{2}$ by Lemma 5. Putting $\gamma_{0}: z(t)=$ $\tilde{G}_{0}^{-1}\left(\sqrt{t} \tilde{G}_{0}\left(z_{0}\right)\right)$, we have $2 \tilde{g}(z(t)) z^{\prime}(t)=\tilde{G}\left(z_{0}\right)$ and

$$
\begin{gathered}
\left\{\tilde{f}_{c}^{\prime}(z(t))-\frac{\alpha+\bar{\alpha}|z(t)|^{2}}{1-|z(t)|^{2}}\right\} z^{\prime}(t) \\
=\frac{c \tilde{G}\left(z_{0}\right)}{\left|\tilde{G}\left(z_{0}\right)\right|} \frac{2(\operatorname{Re} \alpha)|z(t)|}{\left(1-|z(t)|^{2}\right)}\left|z^{\prime}(t)\right| .
\end{gathered}
$$

By integrating the above equality we have

$$
\tilde{f}_{c}\left(z_{0}\right)-\tilde{C}\left(\alpha, \gamma_{0}\right)=\frac{c \tilde{G}\left(z_{0}\right)}{\left|\tilde{G}\left(z_{0}\right)\right|} \tilde{R}\left(\alpha, \gamma_{0}\right)
$$

Since $\tilde{f}_{c}\left(z_{0}\right) \in V_{\mathscr{P}}\left(z_{0}, \alpha\right) \subset \overline{\mathbf{D}}\left(\tilde{C}\left(\alpha, \gamma_{0}\right), \tilde{R}\left(\alpha, \gamma_{0}\right)\right)$ and $\tilde{f}_{c}\left(z_{0}\right) \in \partial \mathbf{D}\left(\tilde{C}\left(\alpha, \gamma_{0}\right), \tilde{R}\left(\alpha, \gamma_{0}\right)\right)$, we have $\tilde{f}_{c}\left(z_{0}\right) \in \partial V_{\mathscr{P}}\left(z_{0}, \alpha\right)$.

Suppose that $f\left(z_{0}\right)=\tilde{f}_{c}\left(z_{0}\right)$ for some $f \in \mathscr{P}(\alpha)$ and $|c|=1$. Put

$$
\tilde{k}(t)=\frac{\left|\tilde{G}\left(z_{0}\right)\right|}{c \tilde{G}\left(z_{0}\right)}\left\{f^{\prime}(z(t))-\frac{\alpha+\bar{\alpha}|z(t)|^{2}}{1-|z(t)|^{2}}\right\} z^{\prime}(t) .
$$

Then we have from (12) and (18)

$$
\operatorname{Re} \tilde{k}(t) \leq|\tilde{k}(t)| \leq \frac{\left|\tilde{G}\left(z_{0}\right)\right|}{c \tilde{G}\left(z_{0}\right)}\left\{\tilde{f}_{c}^{\prime}(z(t))-\frac{\alpha+\bar{\alpha}|z(t)|^{2}}{1-|z(t)|^{2}}\right\} z^{\prime}(t) .
$$

Thus we have from (18), (19) and $f\left(z_{0}\right)=\tilde{f}_{c}\left(z_{0}\right)$

$$
\begin{aligned}
\tilde{R}\left(\alpha, \gamma_{0}\right) & =\operatorname{Re} \tilde{R}\left(\alpha, \gamma_{0}\right) \\
& =\int_{0}^{1} \operatorname{Re} \tilde{k}(t) d t \\
& \leq \int_{0}^{1} \frac{\left|\tilde{G}\left(z_{0}\right)\right|}{c \tilde{G}\left(z_{0}\right)}\left\{\tilde{f}_{c}^{\prime}(z(t))-\frac{\alpha+\bar{\alpha}|z(t)|^{2}}{1-|z(t)|^{2}}\right\} z^{\prime}(t) d t \\
& =\tilde{R}\left(\alpha, \gamma_{0}\right) .
\end{aligned}
$$

Since $\tilde{k}(t)$ is a continuous function of $t \in[0,1]$, this implies $f^{\prime}=\tilde{f}_{c}^{\prime}$ on $\gamma_{0}$ and hence $f=\tilde{f}_{c}$ by normalization.

As in the proof of Theorem 2 it easily follows from uniqueness that the closed curve $\partial \mathbf{D} \ni c \mapsto \tilde{f}_{c}\left(z_{0}\right)$ is simple and coincides with $\partial V_{\mathscr{B}}\left(z_{0}, \alpha\right)$. 
Proof of Corollary 4. From (11) we have

$$
\begin{aligned}
\tilde{f}_{c}\left(z_{0}\right)-i(\operatorname{Im} \alpha) z_{0} & =\operatorname{Re} \alpha \int_{0}^{z_{0}} \frac{1+c \zeta}{1-c \zeta} d \zeta \\
& =(\operatorname{Re} \alpha) z_{0} \int_{0}^{1} \frac{1+c z_{0} t}{1-c z_{0} t} d t .
\end{aligned}
$$

Thus we have

$$
\begin{aligned}
\operatorname{Re}\left(\frac{\tilde{f}_{c}\left(z_{0}\right)}{z_{0}}\right) & =(\operatorname{Re} \alpha) \int_{0}^{1} \operatorname{Re}\left(\frac{1+c z_{0} t}{1-c z_{0} t}\right) d t \\
& =(\operatorname{Re} \alpha) \int_{0}^{1} \frac{1-\left|z_{0}\right|^{2} t^{2}}{\left|1-c z_{0} t\right|^{2}} d t \\
& \geq(\operatorname{Re} \alpha) \int_{0}^{1} \frac{1-\left|z_{0}\right| t}{1+\left|z_{0}\right| t} d t=(\operatorname{Re} \alpha)\left\{\frac{2}{\left|z_{0}\right|} \log \left(1+\left|z_{0}\right|\right)-1\right\}
\end{aligned}
$$

with equality if and only if $c=-\bar{z}_{0} /\left|z_{0}\right|$. From this and Theorem 3 we have for $f \in \mathscr{P}(\alpha)$

$$
\begin{aligned}
\operatorname{Re}\left(\frac{f\left(z_{0}\right)}{z_{0}}\right) & \geq \min _{|c|=1} \operatorname{Re}\left(\frac{\tilde{f}_{c}\left(z_{0}\right)}{z_{0}}\right) \\
& =(\operatorname{Re} \alpha)\left\{\frac{2}{\left|z_{0}\right|} \log \left(1+\left|z_{0}\right|\right)-1\right\}
\end{aligned}
$$

with equality if and only if $f=\tilde{f}_{-\bar{z}_{0} /\left|z_{0}\right|}$.

Since the proof of the other half of the corollary is quite similar, we omit it.

\section{Sufficient conditions for Multivalent starlikeness}

Let $p$ be a positive integer and $\mathscr{A}_{p}$ be the class of analytic functions $f$ in $\mathbf{D}$ with $f(z)=z^{p}+a_{p+1} z^{p+1}+\cdots$.

Lemma 6. Let $f \in \mathscr{A}_{p}$. Then $f \in\left(S^{*}\right)^{p}$ if and only if

$$
\operatorname{Re}\left(z \frac{f^{\prime}(z)}{f(z)}\right)>0, \quad z \in \mathbf{D} .
$$

In the case that $p=1$ the condition (22) implies that $f$ is starlike univalent. When $p \geq 2$, see [5] for a proof.

Proof of Lemma 5. Let $p=1,2, \ldots$ and $f \in \mathscr{A}_{p}$ with $1+\operatorname{Re}\left(z f^{\prime \prime}(z) /\right.$ $\left.f^{\prime}(z)\right)>0$ in D. From Lemma 6 it suffices to prove $\operatorname{Re}\left(z f^{\prime}(z) / f(z)\right)>0$ in $\mathbf{D}$.

First we show that $p^{-1} z f^{\prime}(z) \in\left(S^{*}\right)^{p}$. Since $f^{\prime}(z) \neq 0$ for all $z \in \mathbf{D} \backslash\{0\}$, 
there exists an analytic function $f_{1} \in \mathscr{A}_{1}$ such that $z f^{\prime}(z)=p f_{1}(z)^{p}$ in $\mathbf{D}$. The fact that $f_{1} \in S^{*}$ easily follows from

$$
p \operatorname{Re}\left(z \frac{f_{1}^{\prime}(z)}{f_{1}(z)}\right)=\operatorname{Re}\left(z \frac{f^{\prime \prime}(z)}{f^{\prime}(z)}\right)+1>0 .
$$

For any $z_{1} \in \mathbf{D} \backslash\{0\}$ put $z(t)=f_{1}^{-1}\left(t^{1 / p} f_{1}\left(z_{1}\right)\right), \quad 0 \leq t \leq 1$. Then we have $z(t) f^{\prime}(z(t))=p f_{1}(z(t))^{p}=p t f_{1}\left(z_{1}\right)^{p}=t z_{1} f^{\prime}\left(z_{1}\right)$ and hence

$$
\left\{f^{\prime}(z(t))+z(t) f^{\prime \prime}(z(t))\right\} z^{\prime}(t)=\frac{d}{d t}\left\{z(t) f^{\prime}(z(t))\right\}=z_{1} f^{\prime}\left(z_{1}\right) .
$$

Thus we have for any $z_{1} \in \mathbf{D} \backslash\{0\}$

$$
\begin{aligned}
\operatorname{Re}\left(\frac{f\left(z_{1}\right)}{z_{1} f^{\prime}\left(z_{1}\right)}\right) & =\operatorname{Re}\left\{\int_{0}^{1} \frac{f^{\prime}(z(t)) z^{\prime}(t)}{z_{1} f^{\prime}\left(z_{1}\right)} d t\right\} \\
& =\int_{0}^{1} \operatorname{Re}\left\{\frac{f^{\prime}(z(t))}{f^{\prime}(z(t))+z(t) f^{\prime \prime}(z(t))}\right\} d t \\
& =\int_{0}^{1} \operatorname{Re}\left\{\frac{1}{1+\frac{z(t) f^{\prime \prime}(z(t))}{f^{\prime}(z(t))}}\right\} d t \\
& =\int_{0}^{1}\left\{\frac{1+\operatorname{Re}\left(\frac{z(t) f^{\prime \prime}(z(t))}{f^{\prime}(z(t))}\right)}{\left|1+\frac{z(t) f^{\prime \prime}(z(t))}{f^{\prime}(z(t))}\right|^{2}}\right\} d t>0 .
\end{aligned}
$$

Combining this and $\lim _{z \rightarrow 0} z f^{\prime}(z) / f(z)=p$ we have $\operatorname{Re}\left(z f^{\prime}(z) / f(z)\right)>0$ in $\mathbf{D}$.

Following Goodman [1] let $\mathscr{C}(p)$ be the class of analytic functions $f$ in $\mathbf{D}$ such that there exists $r \in(0,1)$ with the following properties:

(i)

$$
\operatorname{Re}\left(z \frac{f^{\prime \prime}(z)}{f^{\prime}(z)}+1\right)>0 \text { for } r<|z|<1
$$

(ii)

$$
\int_{-\pi}^{\pi} \operatorname{Re}\left(\rho e^{i \theta} \frac{f^{\prime \prime}\left(\rho e^{i \theta}\right)}{f^{\prime}\left(\rho e^{i \theta}\right)}+1\right) d \theta=2 \pi p \quad \text { for } r<\rho<1 .
$$

THeOrem 7. Let $f \in \mathscr{C}(2)$. Then there exists $z_{0} \in \mathbf{D}$ such that $f^{\prime}\left(z_{0}\right)=0$, $f^{\prime \prime}\left(z_{0}\right) \neq 0$ and $f^{\prime}(z) \neq 0$ in $\mathbf{D} \backslash\left\{z_{0}\right\}$, and that

$$
\frac{1}{\left(1-\left|z_{0}\right|^{2}\right)^{2} f^{\prime \prime}\left(z_{0}\right)}\left\{f\left(\frac{z+z_{0}}{1+\overline{z_{0}} z}\right)-f\left(z_{0}\right)\right\} \in\left(S^{*}\right)^{2} \text {. }
$$


Proof. Put

$$
h(z)=z \frac{f^{\prime \prime}(z)}{f^{\prime}(z)}+1, \quad z \in \mathbf{D}
$$

Since for $r<\rho<1$

$$
\begin{aligned}
4 \pi=\int_{0}^{2 \pi} \operatorname{Re}\left\{h\left(\rho e^{i \theta}\right)\right\} d \theta & =\operatorname{Re}\left\{\int_{|z|=\rho}\left(z \frac{f^{\prime \prime}(z)}{f^{\prime}(z)}+1\right) \frac{d z}{i z}\right\} \\
& =2 \pi+\operatorname{Im}\left\{\int_{|z|=\rho} \frac{f^{\prime \prime}(z)}{f^{\prime}(z)} d z\right\},
\end{aligned}
$$

there exists $z_{0} \in \mathbf{D}$ such that $f^{\prime}\left(z_{0}\right)=0, f^{\prime \prime}\left(z_{0}\right) \neq 0$ and $f^{\prime}(z) \neq 0$ for all $z \in$ $\mathbf{D} \backslash\left\{z_{0}\right\}$. Thus the function

$$
g(z)=f\left(\frac{z+z_{0}}{1+\overline{z_{0}} z}\right)-f\left(z_{0}\right), \quad z \in \mathbf{D}
$$

satisfies $g(0)=g^{\prime}(0)=0, g^{\prime \prime}(0)=\left(1-\left|z_{0}\right|^{2}\right)^{2} f^{\prime \prime}\left(z_{0}\right) \neq 0$ and $g^{\prime}(z) \neq 0$ for all $z \in$ $\mathbf{D} \backslash\{0\}$. Hence the function $1+\left(z g^{\prime \prime}(z) / g^{\prime}(z)\right)$ is analytic in $\mathbf{D}$ and its real part is harmonic in $\mathbf{D}$.

We claim that the inequality

$$
\operatorname{Re}\left(z \frac{g^{\prime \prime}(z)}{g^{\prime}(z)}+1\right)>0
$$

holds in $\mathbf{D}$.

From an elementary calculation we have

$$
\begin{aligned}
z \frac{g^{\prime \prime}(z)}{g^{\prime}(z)}+1 & =1-\frac{2 \overline{z_{0}} z}{1+\overline{z_{0}} z}+\frac{\left(1-\left|z_{0}\right|^{2}\right) z}{\left(1+\overline{z_{0}} z\right)^{2}} \frac{f^{\prime \prime}\left(\frac{z+z_{0}}{1+\overline{z_{0}} z}\right)}{f^{\prime}\left(\frac{z+z_{0}}{1+\overline{z_{0}} z}\right)} \\
& =\frac{z_{0}-\overline{z_{0}} z^{2}}{\left(z+z_{0}\right)\left(1+\overline{z_{0}} z\right)}+\frac{\left(1-\left|z_{0}\right|^{2}\right) z}{\left(z+z_{0}\right)\left(1+\overline{z_{0}} z\right)} h\left(\frac{z+z_{0}}{1+\overline{z_{0}} z}\right) .
\end{aligned}
$$

Without loss of generality we may assume $h$ is continuous on $\overline{\mathbf{D}}$. Since for $|z|=1$ we have from the identities

$$
\begin{aligned}
& \frac{z_{0}-\overline{z_{0}} z^{2}}{\left(z+z_{0}\right)\left(1+\overline{z_{0}} z\right)}=-2 i \frac{\operatorname{Im}\left(\overline{z_{0}} z\right)}{\left|z+z_{0}\right|^{2}}, \\
& \frac{\left(1-\left|z_{0}\right|^{2}\right) z}{\left(z+z_{0}\right)\left(1+\overline{z_{0}} z\right)}=\frac{1-\left|z_{0}\right|^{2}}{\left|z+z_{0}\right|^{2}},
\end{aligned}
$$

it follows that 


$$
\liminf _{\mathbf{D} \ni z \rightarrow \zeta} \operatorname{Re}\left(z \frac{g^{\prime \prime}(z)}{g^{\prime}(z)}+1\right) \geq 0
$$

for all $\zeta \in \partial \mathbf{D}$. From this and $\lim _{z \rightarrow 0} z g^{\prime}(z) / g(z)=1$ we have $\operatorname{Re}\left(z g^{\prime \prime}(z) / g^{\prime}(z)\right)+$ $1>0$ in D.

5.

Now the fact that $2\left(1-\left|z_{0}\right|^{2}\right)^{-2} f^{\prime \prime}\left(z_{0}\right)^{-1} g \in\left(S^{*}\right)^{2}$ easily follows from Lemma

\section{REFERENCES}

[1] A. W. Goodman, On the Schwarz-Christoffel transformation and p-valent functions, Trans. Amer. Math. Soc. 68 (1950), 204-223.

[2] A. W. Goodman, Univalent Functions I and II, Mariner Publishing Co., Tampa, Florida, 1983.

[ 3 ] D. J. Hallenbeck and A. E. Livingston, Applications of extreme point theory to classes of multivalent functions, Trans. Amer. Math. Soc. 221 (1976), 339-359.

[ 4 ] R. J. Libera, Some classes of regular univalent functions, Proc. of the Amer. Math. Soc. 16 (1965), 755-758.

[5] M. S. Robertson, The partial sums of multivalently star-like functions, Ann. of math. 42 (1941), 829-838.

Department of Applied Science

FACULTY OF ENGINEERING

YAMAGUCHI UNIVERSITY

ToKIWADAI 2-16-1, UBE, 755-8611

JAPAN

e-mail: hiroshi@yamaguchi-u.ac.jp 